\title{
Noninstantaneous impulsive inequalities via conformable fractional calculus
}

\author{
Surang Sitho', Sotiris K. Ntouyas ${ }^{2,3}$, Praveen Agarwal ${ }^{4}$ and Jessada Tariboon ${ }^{5 *}$ [D
}

\author{
*Correspondence: \\ jessada.t@sci.kmutnb.ac.th \\ ${ }^{5}$ Intelligent and Nonlinear Dynamic \\ Innovations Research Center, \\ Department of Mathematics, \\ Faculty of Applied Science, King \\ Mongkut's University of Technology \\ North Bangkok, Bangkok, Thailand \\ Full list of author information is \\ available at the end of the article
}

\author{
Abstract \\ We establish some new noninstantaneous impulsive inequalities using the \\ conformable fractional calculus. \\ MSC: 26D10; 26A33; 34A37 \\ Keywords: Conformable fractional derivative; Conformable fractional integral; \\ Impulsive inequalities
}

\section{Introduction and preliminaries}

The subject of fractional differential equations has evolved as an interesting and important field of research in view of numerous applications in physics, mechanics, chemistry, engineering (like traffic, transportation, logistic, etc.), and so forth [1-3]. The tools of fractional calculus play a key role in improving the mathematical modeling of many real-world processes based on classical calculus. For some recent development on the topic, see [412] and the references therein.

Various types of fractional derivatives were introduced: Riemann-Liouville, Caputo, Hadamard, Erdélyi-Kober, Grünwald-Letnikov, Marchaud, and Riesz, to just name a few. Commonly, all they are defined as integrals with different singular kernels, that is, they have a nonlocal structure. Due to this fact, there are many inconsistencies of the existing fractional derivatives with classical derivative. Thus they do not obey the familiar product rule, the quotient rule for two functions, and the chain rule. Also, the fractional derivatives do not have a corresponding Rolle's theorem or a corresponding mean value theorem.

On the other hand, a recently introduced definition of the so-called conformable fractional derivative involves a limit instead of an integral; see [13, 14]. This local definition enables us to prove many properties analogous to those of integer-order derivatives. The authors in [14] showed that the conformable fractional derivative obeys the product and quotient rules and has results similar to the Rolle theorem and the mean value theorem in classical calculus.

For recent works on conformable derivatives, we refer to [15-19] and the references therein.

Let us recall the definition of the conformable fractional derivative and integral.

(c) The Author(s) 2018. This article is distributed under the terms of the Creative Commons Attribution 4.0 International License (http://creativecommons.org/licenses/by/4.0/), which permits unrestricted use, distribution, and reproduction in any medium, provided you give appropriate credit to the original author(s) and the source, provide a link to the Creative Commons license, and indicate if changes were made. 
Definition 1.1 Let $0<\alpha \leq 1$. The conformable fractional derivative starting from a point $\phi$ of a function $f:[\phi, \infty) \rightarrow \mathbb{R}$ is defined by

$$
{ }_{\phi} D^{\alpha}(t)=\lim _{\epsilon \rightarrow 0} \frac{f\left(t+\epsilon(t-\phi)^{1-\alpha}\right)-f(t)}{\epsilon}, \quad t>\phi,
$$

and ${ }_{\phi} D^{\alpha} f(\phi)=\lim _{t \rightarrow \phi^{+} \phi} D^{\alpha} f(t)$.

Note that if $f$ is differentiable, then

$$
{ }_{\phi} D^{\alpha} f(t)=(t-\phi)^{1-\alpha} \frac{d f(t)}{d t} .
$$

Definition 1.2 Let $0<\alpha \leq 1$. The conformable fractional integral of a function $f$ : $[\phi, \infty) \rightarrow \mathbb{R}$ from a point $\phi$ is defined by

$$
{ }_{\phi} I^{\alpha}(t)=\int_{\phi}^{t}(s-\phi)^{\alpha-1} f(s) d s
$$

The impulsive differential equations have been used to describe processes that have sudden changes in their states at certain moments. Many mathematical models in physical phenomena that have short-term perturbations at fixed impulse points $t_{k}, k=1,2,3, \ldots$, caused by external interventions during their evolution appeared in population dynamics, biotechnology processes, chemistry, physics, engineering, and medicine; see [20-22]. In [23, 24], the authors introduced a new class of noninstantaneous impulsive differential equations with initial conditions to describe some certain dynamic changes of evolution processes in the pharmacotherapy. This kind of impulsive differential equations can be distinguished from the usual one as the changing processes containing no ordinary or fractional derivatives of their states work over intervals $\left(t_{k}, s_{k}\right]$, whereas the usual does at points $t_{k}, k=1,2,3, \ldots$. There are some papers on existence and stability theory of this kind of impulsive ordinary or fractional differential equations [25-36]. To the best of our knowledge, there is no literature on noninstantaneous impulsive inequalities. The main goal of the paper is to establish some new noninstantaneous impulsive inequalities using the conformable fractional calculus. The main results are presented in Sect. 2. In Sect. 3, the maximum principle and boundedness of solutions for noninstantaneous impulse problems are illustrated.

\section{Main results}

Assume that the independent variable $t$ is the time defined on the half-line $\mathbb{R}_{+}=[0, \infty)$. Let $\left\{t_{i}\right\}_{i=1}^{\infty}$ and $\left\{s_{i}\right\}_{i=0}^{\infty}$ be two increasing sequences such that

$$
0=s_{0}<t_{1} \leq s_{1}<t_{2} \leq s_{2}<t_{3} \leq \cdots<t_{i} \leq s_{i}<t_{i+1} \leq \cdots
$$

for $i=1,2, \ldots$ and $\lim _{k \rightarrow \infty} t_{k}=\lim _{k \rightarrow \infty} s_{k}=\infty$. In addition, we define subsets of $\mathbb{R}_{+}$by $U_{s_{k}}=\bigcup_{k=0}^{\infty}\left(s_{k}, t_{k+1}\right], U_{t_{k}}=\bigcup_{k=1}^{\infty}\left(t_{k}, s_{k}\right]$ and $U=U_{s_{k}} \cup U_{t_{k}}$. Note that $U \cup\{0\}=\mathbb{R}_{+}$. Set $P C\left(U_{s_{k}}, \mathbb{R}\right)=\left\{x: U_{s_{k}} \rightarrow \mathbb{R} ; x(t)\right.$ is continuous on $U_{s_{k}}$, and $x\left(s_{k}^{+}\right)$exists for $\left.k=0,1,2, \ldots\right\}$, $P C\left(U_{t_{k}}, \mathbb{R}\right)=\left\{x: U_{t_{k}} \rightarrow \mathbb{R} ; x(t)\right.$ is continuous for $t \in U_{t_{k}}$, and $x\left(t_{k}^{+}\right)$exists for $\left.k=1,2,3, \ldots\right\}$, $P C_{s_{k}}^{\alpha}\left(U_{s_{k}}, \mathbb{R}\right)=\left\{x \in P C\left(U_{s_{k}}, \mathbb{R}\right):_{s_{k}} D^{\alpha} x(t)\right.$ is continuous everywhere for $t \in U_{s_{k}}$, and 
${ }_{s_{k}} D^{\alpha} x\left(s_{k}^{+}\right)$exists for $\left.k=0,1,2, \ldots\right\}, P C_{t_{k}}^{\alpha}\left(U_{t_{k}}, \mathbb{R}\right)=\left\{x \in P C\left(U_{t_{k}}, \mathbb{R}\right):{ }_{t_{k}} I^{\alpha} x(t)\right.$ is continuous everywhere for all $t \in U_{t_{k}}$, and $t_{k} I^{\alpha} x\left(t_{k}^{+}\right)$exists for $\left.k=1,2,3, \ldots\right\}$, and $P C^{\alpha}(U, \mathbb{R})=$ $P C_{s_{k}}^{\alpha}\left(U_{s_{k}}, \mathbb{R}\right) \cup P C_{t_{k}}^{\alpha}\left(U_{t_{k}}, \mathbb{R}\right)$.

Let the maximums of impulsive points less than or equal to $t$ be defined by

$$
s_{m}=\max \left\{s_{k}: s_{k} \leq t, k=0,1,2, \ldots\right\} \quad \text { and } \quad t_{\bar{m}}=\max \left\{t_{k}: t_{k} \leq t, k=1,2,3, \ldots\right\} .
$$

In addition, we define

$$
\begin{aligned}
& H_{k}=e^{d_{k} \frac{\left(s_{k}-t_{k}\right)^{\alpha}}{\alpha}} ; \quad Q_{k}=e^{\int_{s_{k-1}}^{t_{k}} p(\xi)\left(\xi-s_{k-1}\right)^{\alpha-1} d \xi} ; \\
& G_{k}=Q_{k} H_{k} ; \quad P_{k}=Q_{k} H_{k-1} .
\end{aligned}
$$

Note that

$$
H_{m} G_{m+1} G_{m+2} G_{m+3} \cdots G_{n-1} Q_{n}=P_{m+1} P_{m+2} \cdots P_{n-1} P_{n}
$$

and

$$
P_{m} P_{m+1} P_{m+2} \cdots P_{n-1} P_{n} H_{n}=H_{m-1} G_{m} G_{m+1} \cdots G_{n-1} G_{n}
$$

where $m<n$ are positive integers.

Throughout this paper, we assume that the unknown function $u \in P C^{\alpha}(U, \mathbb{R})$ is leftcontinuous at $s_{k}$ and $t_{k}(k=1,2,3, \ldots)$. Now, we are in the position to establish noninstantaneous impulsive differential inequalities.

Theorem 2.1 Let $b_{k}, c_{k}, d_{k}$ be given constants such that $b_{k}, c_{k} \geq 0$ and $d_{k}>0, k=1,2,3, \ldots$. Suppose that $p, q \in P C\left(U_{s_{k}}, \mathbb{R}\right)$ and

$$
\left\{\begin{array}{l}
s_{k} D^{\alpha} u(t) \leq p(t) u(t)+q(t), \quad t \in\left(s_{k}, t_{k+1}\right], k=0,1,2, \ldots \\
u(t) \leq c_{k} u\left(t_{k}^{-}\right)+d_{k} \int_{t_{k}}^{t}\left(\xi-t_{k}\right)^{\alpha-1} u(\xi) d \xi+b_{k}, \quad t \in\left(t_{k}, s_{k}\right], k=1,2, \ldots
\end{array}\right.
$$

Then

$$
\begin{aligned}
u(t) \leq & e^{\int_{s_{m}}^{t} p(\xi)\left(\xi-s_{m}\right)^{\alpha-1} d \xi}\left\{u\left(s_{0}\right) \prod_{0<k \leq m} c_{k} G_{k}+\sum_{0<k \leq m}\left(\prod_{k<j \leq m} c_{j} G_{j}\right) H_{k} b_{k}\right. \\
& \left.+\sum_{0<k \leq m}\left(\prod_{k<j \leq m} c_{j} G_{j}\right) c_{k} H_{k} \int_{s_{k-1}}^{t_{k}} q(\eta)\left(\eta-s_{k-1}\right)^{\alpha-1} e^{\int_{\eta}^{t_{k}} p(\xi)\left(\xi-s_{k-1}\right)^{\alpha-1} d \xi} d \eta\right\} \\
& +\int_{s_{m}}^{t} q(\eta)\left(\eta-s_{m}\right)^{\alpha-1} e^{\int_{\eta}^{t} p(\xi)\left(\xi-s_{m}\right)^{\alpha-1} d \xi} d \eta, \quad t \in\left(s_{k}, t_{k+1}\right], k=0,1,2, \ldots,
\end{aligned}
$$

and

$$
\begin{aligned}
& u(t) \leq e^{d_{\bar{m}} \frac{\left(t-t_{\bar{m})^{\alpha}}\right)^{\alpha}}{\alpha}}\left\{u\left(s_{0}\right) c_{\bar{m}} Q_{\bar{m}} \prod_{0<k<\bar{m}} c_{k} G_{k}+\sum_{0<k \leq \bar{m}}\left(\prod_{k<j \leq \bar{m}} c_{j} P_{j}\right) b_{k}\right. \\
& \left.+\sum_{0<k \leq \bar{m}}\left(\prod_{k<j \leq \bar{m}} c_{j} P_{j}\right) c_{k} \int_{s_{k-1}}^{t_{k}} q(\eta)\left(\eta-s_{k-1}\right)^{\alpha-1} e^{\int_{\eta}^{t_{k}} p(\xi)\left(\xi-s_{k-1}\right)^{\alpha-1} d \xi} d \eta\right\}, \\
& t \in\left(t_{k}, s_{k}\right], k=1,2,3, \ldots .
\end{aligned}
$$


Proof For $t \in\left(s_{0}, t_{1}\right]$, the conformable fractional differential inequality can be written as

$$
{ }_{s_{0}} D^{\alpha}\left[u(t) e^{-\int_{s_{0}}^{t} p(\xi)\left(\xi-s_{0}\right)^{\alpha-1} d \xi}\right] \leq q(t) e^{-\int_{s_{0}}^{t} p(\xi)\left(\xi-s_{0}\right)^{\alpha-1} d \xi} .
$$

By taking the conformable fractional integral of order $\alpha$ from $s_{0}$ to $t \in\left(s_{0}, t_{1}\right]$,

$$
{ }_{s_{0}} I^{\alpha}{ }_{{ }_{0}} D^{\alpha}\left[u(t) e^{-\int_{s_{0}}^{t} p(\xi)\left(\xi-s_{0}\right)^{\alpha-1} d \xi}\right] \leq{ }_{s_{0}} I^{\alpha}\left[q(t) e^{-\int_{s_{0}}^{t} p(\xi)\left(\xi-s_{0}\right)^{\alpha-1} d \xi}\right],
$$

we obtain

$$
\begin{aligned}
& u(t) \leq u\left(s_{0}\right) e^{\int_{s_{0}}^{t} p(\xi)\left(\xi-s_{0}\right)^{\alpha-1} d \xi} \\
& +\int_{s_{0}}^{t} q(\eta)\left(\eta-s_{0}\right)^{\alpha-1} e^{\int_{\eta}^{t} p(\xi)\left(\xi-s_{0}\right)^{\alpha-1} d \xi} d \eta, \quad t \in\left(s_{0}, t_{1}\right],
\end{aligned}
$$

which implies that (2.5) holds for $k=0$.

For $t \in\left(t_{1}, s_{1}\right]$, we define the function

$$
z(t)=\int_{t_{1}}^{t}\left(\xi-t_{1}\right)^{\alpha-1} u(\xi) d \xi
$$

Note that $z\left(t_{1}\right)=0$ and

$$
u(t) \leq c_{1} u\left(t_{1}^{-}\right)+d_{1} z(t)+b_{1}, \quad t \in\left(t_{1}, s_{1}\right] .
$$

Then, taking the derivative with respect to $t$, we have

$$
\begin{aligned}
z^{\prime}(t) & =\left(t-t_{1}\right)^{\alpha-1} u(t) \\
& \leq\left(t-t_{1}\right)^{\alpha-1}\left[c_{1} u\left(t_{1}^{-}\right)+b_{1}\right]+d_{1}\left(t-t_{1}\right)^{\alpha-1} z(t) .
\end{aligned}
$$

Multiplying this inequality by the integrating factor $e^{-d_{1} \frac{\left(t-t_{1}\right)^{\alpha}}{\alpha}}$, we get

$$
\frac{d}{d t}\left[z(t) e^{-d_{1} \frac{\left(t-t_{1}\right)^{\alpha}}{\alpha}}\right] \leq\left[c_{1} u\left(t_{1}^{-}\right)+b_{1}\right]\left(t-t_{1}\right)^{\alpha-1} e^{-d_{1} \frac{\left(t-t_{1}\right)^{\alpha}}{\alpha}},
$$

which implies that

$$
\begin{aligned}
z(t) & \leq\left[c_{1} u\left(t_{1}^{-}\right)+b_{1}\right] e^{d_{1} \frac{\left(t-t_{1}\right)^{\alpha}}{\alpha}} \int_{t_{1}}^{t}\left(\eta-t_{1}\right)^{\alpha-1} e^{-d_{1} \frac{\left(\eta-t_{1}\right)^{\alpha}}{\alpha}} d \eta \\
& =\frac{1}{d_{1}}\left[c_{1} u\left(t_{1}^{-}\right)+b_{1}\right]\left[e^{d_{1} \frac{\left(t-t_{1}\right)^{\alpha}}{\alpha}}-1\right] .
\end{aligned}
$$

By (2.7) with $t=t_{1}$ we have

$$
\begin{aligned}
u(t) \leq & c_{1} e^{d_{1} \frac{\left(t-t_{1}\right)^{\alpha}}{\alpha}}\left[u\left(s_{0}\right) e^{\int_{s_{0}}^{t_{1}} p(\xi)\left(\xi-s_{0}\right)^{\alpha-1} d \xi}\right. \\
& \left.+\int_{s_{0}}^{t_{1}} q(\eta)\left(\eta-s_{0}\right)^{\alpha-1} e^{\int_{\eta}^{t_{1}} p(\xi)\left(\xi-s_{0}\right)^{\alpha-1} d \xi} d \eta\right]+b_{1} e^{d_{1} \frac{\left(t-t_{1}\right)^{\alpha}}{\alpha}}, \quad t \in\left(t_{1}, s_{1}\right] .
\end{aligned}
$$

This shows that the bound in (2.6) is true for $k=1$. 
Now, we assume that inequality (2.5) holds for $t \in\left(s_{n}, t_{n+1}\right], n>0$. By mathematical induction we will show that (2.6) is true for $t \in\left(t_{n+1}, s_{n+1}\right]$. Let

$$
w(t)=\int_{t_{n+1}}^{t}\left(\xi-t_{n+1}\right)^{\alpha-1} u(\xi) d \xi, \quad t \in\left(t_{n+1}, s_{n+1}\right] .
$$

Then $w\left(t_{n+1}\right)=0$ and $u(t) \leq c_{n+1} u\left(t_{n+1}^{-}\right)+d_{n+1} w(t)+b_{n+1}$. Using the above method, we have

$$
w^{\prime}(t) \leq\left(t-t_{n+1}\right)^{\alpha-1}\left[c_{n+1} u\left(t_{n+1}^{-}\right)+b_{n+1}\right]+d_{n+1}\left(t-t_{n+1}\right)^{\alpha-1} w(t)
$$

which leads to

$$
w(t) \leq \frac{1}{d_{n+1}}\left[c_{n+1} u\left(t_{n+1}^{-}\right)+b_{n+1}\right]\left[e^{d_{n+1} \frac{\left(t-t_{n+1}\right)^{\alpha}}{\alpha}}-1\right] .
$$

Substituting the bound of $w(t)$ and inequality (2.5) with $t=t_{n+1}$, it follows that

$$
\begin{aligned}
& u(t) \leq c_{n+1} u\left(t_{n+1}^{-}\right)+d_{n+1} w(t)+b_{n+1} \\
& \leq c_{n+1} u\left(t_{n+1}^{-}\right) e^{d_{n+1} \frac{\left(t-t_{n+1}\right)^{\alpha}}{\alpha}}+b_{n+1} e^{d_{n+1} \frac{\left(t-t_{n+1}\right)^{\alpha}}{\alpha}} \\
& \leq c_{n+1}\left[e ^ { \int _ { s _ { n } } ^ { t _ { n + 1 } } p ( \xi ) ( \xi - s _ { n } ) ^ { \alpha - 1 } d \xi } \left\{u\left(s_{0}\right) \prod_{0<k \leq n} c_{k} G_{k}+\sum_{0<k \leq n}\left(\prod_{k<j \leq n} c_{j} G_{j}\right) H_{k} b_{k}\right.\right. \\
& \left.+\sum_{0<k \leq n}\left(\prod_{k<j \leq n} c_{j} G_{j}\right) c_{k} H_{k} \int_{s_{k-1}}^{t_{k}} q(\eta)\left(\eta-s_{k-1}\right)^{\alpha-1} e^{\int_{\eta}^{t_{k}} p(\xi)\left(\xi-s_{k-1}\right)^{\alpha-1} d \xi} d \eta\right\} \\
& \left.+\int_{s_{n}}^{t_{n+1}} q(\eta)\left(\eta-s_{n}\right)^{\alpha-1} e^{\int_{\eta}^{t_{n+1}} p(\xi)\left(\xi-s_{n}\right)^{\alpha-1} d \xi} d \eta\right] e^{d_{n+1} \frac{\left(t-t_{n+1}\right)^{\alpha}}{\alpha}} \\
& +b_{n+1} e^{d_{n+1} \frac{\left(t-t_{n+1}\right)^{\alpha}}{\alpha}} \\
& =e^{d_{n+1} \frac{\left(t-t_{n+1}\right)^{\alpha}}{\alpha}}\left\{u\left(s_{0}\right) c_{n+1} Q_{n+1} \prod_{0<k<n+1} c_{k} G_{k}+\sum_{0<k \leq n+1}\left(\prod_{k<j \leq n+1} c_{j} P_{j}\right) b_{k}\right. \\
& \left.+\sum_{0<k \leq n+1}\left(\prod_{k<j \leq n+1} c_{j} P_{j}\right) c_{k} \int_{s_{k-1}}^{t_{k}} q(\eta)\left(\eta-s_{k-1}\right)^{\alpha-1} e^{\int_{\eta}^{t_{k}} p(\xi)\left(\xi-s_{k-1}\right)^{\alpha-1} d \xi} d \eta\right\}
\end{aligned}
$$

by using formula (2.2). Therefore (2.6) is satisfied for $t \in\left(t_{n+1}, s_{n+1}\right]$.

Finally, we suppose that estimate (2.6) is fulfilled for $t \in\left(t_{n}, s_{n}\right]$, where $n>1$. Next, we will prove that inequality $(2.5)$ holds for $\left(s_{n}, t_{n+1}\right]$. By using the above method, we get the inequality

$$
\begin{aligned}
u(t) \leq & u\left(s_{n}\right) e^{\int_{s_{n}}^{t} p(\xi)\left(\xi-s_{n}\right)^{\alpha-1} d \xi} \\
& +\int_{s_{n}}^{t} q(\eta)\left(\eta-s_{n}\right)^{\alpha-1} e^{\int_{\eta}^{t} p(\xi)\left(\xi-s_{n}\right)^{\alpha-1} d \xi} d \eta, \quad t \in\left(s_{n}, t_{n+1}\right] .
\end{aligned}
$$


Using (2.6) with $t=s_{n}$ and applying (2.3), we obtain

$$
\begin{aligned}
u(t) \leq & e^{\int_{s_{n}}^{t} p(\xi)\left(\xi-s_{n}\right)^{\alpha-1} d \xi}\left[e ^ { d _ { n } \frac { ( s _ { n } - t _ { n } ) ^ { \alpha } } { \alpha } } \left\{u\left(s_{0}\right) c_{n} Q_{n} \prod_{0<k<n} c_{k} G_{k}+\sum_{0<k \leq n}\left(\prod_{k<j \leq n} c_{j} P_{j}\right) b_{k}\right.\right. \\
& \left.\left.+\sum_{0<k \leq n}\left(\prod_{k<j \leq n} c_{j} P_{j}\right) c_{k} \int_{s_{k-1}}^{t_{k}} q(\eta)\left(\eta-s_{k-1}\right)^{\alpha-1} e^{\int_{\eta}^{t_{k}} p(\xi)\left(\xi-s_{k-1}\right)^{\alpha-1} d \xi} d \eta\right\}\right] \\
& +\int_{s_{n}}^{t} q(\eta)\left(\eta-s_{n}\right)^{\alpha-1} e^{\int_{\eta}^{t} p(\xi)\left(\xi-s_{n}\right)^{\alpha-1} d \xi} d \eta \\
= & e^{\int_{s_{n}}^{t} p(\xi)\left(\xi-s_{n}\right)^{\alpha-1} d \xi}\left[u\left(s_{0}\right) \prod_{0<k \leq n} c_{k} G_{k}+\sum_{0<k \leq n}\left(\prod_{k<j \leq n} c_{j} G_{j}\right) H_{k} b_{k}\right. \\
& \left.+\sum_{0<k \leq n}\left(\prod_{k<j \leq n} c_{j} G_{j}\right) c_{k} H_{k} \int_{s_{k-1}}^{t_{k}} q(\eta)\left(\eta-s_{k-1}\right)^{\alpha-1} e^{\int_{\eta}^{t_{k}} p(\xi)\left(\xi-s_{k-1}\right)^{\alpha-1} d \xi} d \eta\right] \\
& +\int_{s_{n}}^{t} q(\eta)\left(\eta-s_{n}\right)^{\alpha-1} e^{\int_{\eta}^{t} p(\xi)\left(\xi-s_{n}\right)^{\alpha-1} d \xi} d \eta .
\end{aligned}
$$

Therefore inequality $(2.5)$ is valid on $\left(s_{n}, t_{n+1}\right]$. This completes the proof.

The following corollary can be obtained by replacing the given functions $p(t)$ and $q(t)$ by constants $M$ and $N$, respectively.

Corollary 2.1 Let $b_{k}, c_{k} \geq 0$ and $d_{k}>0, k=1,2,3, \ldots$, be constants. If $M>0, N \in \mathbb{R}$, and

$$
\left\{\begin{array}{l}
{ }_{s_{k}} D^{\alpha} u(t) \leq M u(t)+N, \quad t \in\left(s_{k}, t_{k+1}\right], k=0,1,2, \ldots, \\
u(t) \leq c_{k} u\left(t_{k}^{-}\right)+d_{k} \int_{t_{k}}^{t}\left(\xi-t_{k}\right)^{\alpha-1} u(\xi) d \xi+b_{k}, \quad t \in\left(t_{k}, s_{k}\right], k=1,2, \ldots
\end{array}\right.
$$

then

$$
\begin{aligned}
u(t) \leq & e^{M \frac{\left(t-s_{m}\right)^{\alpha}}{\alpha}}\left\{u\left(s_{0}\right) \prod_{0<k \leq m} c_{k} G_{k}^{*}+\sum_{0<k \leq m}\left(\prod_{k<j \leq m} c_{j} G_{j}^{*}\right) H_{k} b_{k}\right. \\
& \left.+\frac{N}{M} \sum_{0<k \leq m}\left(\prod_{k<j \leq m} c_{j} G_{j}^{*}\right) c_{k} H_{k}\left(e^{M \frac{\left(t_{k}-s_{k-1}\right)^{\alpha}}{\alpha}}-1\right)\right\} \\
& +\frac{N}{M}\left(e^{M \frac{\left(t-s_{m}\right)^{\alpha}}{\alpha}}-1\right), \quad t \in\left(s_{k}, t_{k+1}\right], k=0,1,2, \ldots,
\end{aligned}
$$

and

$$
\begin{aligned}
u(t) \leq & e^{d_{\bar{m}} \frac{\left(t-t_{\bar{m}}\right)^{\alpha}}{\alpha}}\left\{u\left(s_{0}\right) c_{\bar{m}} Q_{\bar{m}}^{*} \prod_{0<k<\bar{m}} c_{k} G_{k}^{*}+\sum_{0<k \leq \bar{m}}\left(\prod_{k<j \leq \bar{m}} c_{j} P_{j}^{*}\right) b_{k}\right. \\
& \left.+\frac{N}{M} \sum_{0<k \leq \bar{m}}\left(\prod_{k<j \leq \bar{m}} c_{j} P_{j}^{*}\right) c_{k}\left(e^{M \frac{\left(t_{k}-s_{k-1}\right)^{\alpha}}{\alpha}}-1\right)\right\}, \\
& t \in\left(t_{k}, s_{k}\right], k=1,2,3, \ldots,
\end{aligned}
$$

where $Q_{k}^{*}=e^{M \frac{\left(t_{k}-s_{k-1}\right)^{\alpha}}{\alpha}}, G_{k}^{*}=Q_{k}^{*} H_{k}$, and $P_{k}^{*}=Q_{k}^{*} H_{k-1}$. 
Let $H(t)$ be the Heaviside function. We define two functions

$$
\begin{aligned}
\varphi(t) & =\sum_{i=0}^{\infty} H\left(t-s_{i}\right)-H\left(t-t_{i+1}^{+}\right) \\
& = \begin{cases}0, & t \in\left(t_{k}, s_{k}\right], k=1,2,3, \ldots, \\
1, & t \in\left(s_{k}, t_{k+1}\right], k=0,1,2, \ldots,\end{cases}
\end{aligned}
$$

and

$$
\begin{aligned}
\psi(t) & =\sum_{i=1}^{\infty} H\left(t-t_{i}\right)-H\left(t-s_{i}^{+}\right) \\
& = \begin{cases}0, & t \in\left(s_{k}, t_{k+1}\right], k=0,1,2, \ldots \\
1, & t \in\left(t_{k}, s_{k}\right], k=1,2,3, \ldots\end{cases}
\end{aligned}
$$

Next, we establish some new noninstantaneous impulsive integral inequalities.

Theorem 2.2 Let $p \in P C\left(U_{s_{k}}, \mathbb{R}_{+}\right)$, constants $c_{k}, b_{k} \geq 0, d_{k}>0, k=1,2,3, \ldots$, and $A \in \mathbb{R}$. If

$$
\begin{aligned}
u(t) \leq & \left(A+\int_{s_{m}}^{t}\left(\xi-s_{m}\right)^{\alpha-1} p(\xi) u(\xi) d \xi\right) \varphi(t) \\
& +\left(c_{\bar{m}} u\left(t_{\bar{m}}^{-}\right)+d_{\bar{m}} \int_{t_{\bar{m}}}^{t}\left(\xi-t_{\bar{m}}\right)^{\alpha-1} u(\xi) d \xi+b_{\bar{m}}\right) \psi(t), \quad t \in \mathbb{R}_{+},
\end{aligned}
$$

where $s_{m}$ and $t_{\bar{m}}$ are defined by (2.1), then we have

$$
u(t) \leq e^{\int_{s_{m}}^{t} p(\xi)\left(\xi-s_{m}\right)^{\alpha-1} d \xi}\left(A \prod_{0<k \leq m} c_{k} G_{k}+\sum_{0<k \leq m}\left(\prod_{k<j \leq m} c_{j} G_{j}\right) H_{k} b_{k}\right)
$$

for $t \in\left(s_{k}, t_{k+1}\right], k=0,1,2, \ldots$, and

$$
u(t) \leq e^{d_{\bar{m}} \frac{\left(t-t_{\bar{m})^{\alpha}}^{\alpha}\right.}{\alpha}}\left(A c_{\bar{m}} Q_{\bar{m}} \prod_{0<k<\bar{m}} c_{k} G_{k}+\sum_{0<k \leq \bar{m}}\left(\prod_{k<j \leq \bar{m}} c_{j} P_{j}\right) b_{k}\right)
$$

for $t \in\left(t_{k}, s_{k}\right], k=1,2,3, \ldots$

Proof To prove inequalities (2.13) and (2.14), for $t \in \mathbb{R}_{+}$, we define the function

$$
\begin{aligned}
v(t)= & \left(A+\int_{s_{m}}^{t}\left(\xi-s_{m}\right)^{\alpha-1} p(\xi) u(\xi) d \xi\right) \varphi(t) \\
& +\left(c_{\bar{m}} u\left(t_{\bar{m}}^{-}\right)+d_{\bar{m}} \int_{t_{\bar{m}}}^{t}\left(\xi-t_{\bar{m}}\right)^{\alpha-1} u(\xi) d \xi+b_{\bar{m}}\right) \psi(t),
\end{aligned}
$$

which yields $u(t) \leq v(t)$ for all $t \in \mathbb{R}_{+}$and $v\left(s_{0}\right)=A$. For any $t \in\left(s_{k}, t_{k+1}\right], k=0,1,2, \ldots$, we get

$$
v(t)=A+\int_{s_{m}}^{t}\left(\xi-s_{m}\right)^{\alpha-1} p(\xi) u(\xi) d \xi
$$


Also, taking the conformable fractional derivative of order $\alpha$, we have

$$
{ }_{s_{m}} D^{\alpha} v(t)=p(t) u(t) \leq p(t) v(t) .
$$

For $t \in\left(t_{k}, s_{k}\right], k=1,2,3, \ldots$, we obtain

$$
\begin{aligned}
v(t) & =c_{\bar{m}} u\left(t_{\bar{m}}^{-}\right)+d_{\bar{m}} \int_{t_{\bar{m}}}^{t}\left(\xi-t_{\bar{m}}\right)^{\alpha-1} u(\xi) d \xi+b_{\bar{m}} \\
& \leq c_{\bar{m}} v\left(t_{\bar{m}}^{-}\right)+d_{\bar{m}} \int_{t_{\bar{m}}}^{t}\left(\xi-t_{\bar{m}}\right)^{\alpha-1} v(\xi) d \xi+b_{\bar{m}} .
\end{aligned}
$$

An application of Theorem 2.1 to (2.15) and (2.16) yields

$$
v(t) \leq e^{\int_{s_{m}}^{t} p(\xi)\left(\xi-s_{m}\right)^{\alpha-1} d \xi}\left(A \prod_{0<k \leq m} c_{k} G_{k}+\sum_{0<k \leq m}\left(\prod_{k<j \leq m} c_{j} G_{j}\right) H_{k} b_{k}\right)
$$

for $t \in\left(s_{k}, t_{k+1}\right], k=0,1,2, \ldots$, and

$$
v(t) \leq e^{d_{\bar{m}} \frac{\left(t-t_{\bar{m}}\right)^{\alpha}}{\alpha}}\left(A c_{\bar{m}} Q_{\bar{m}} \prod_{0<k<\bar{m}} c_{k} G_{k}+\sum_{0<k \leq \bar{m}}\left(\prod_{k<j \leq \bar{m}} c_{j} P_{j}\right) b_{k}\right)
$$

for $t \in\left(t_{k}, s_{k}\right], k=1,2,3, \ldots$. From $u(t) \leq v(t), t \in \mathbb{R}_{+}$, we get the desired results in (2.13) and (2.14). The proof is completed.

Theorem 2.3 Let $p \in P C\left(U_{s_{k}}, \mathbb{R}_{+}\right)$, let h be a positive fractional integrable function of order $\alpha$, and let $c_{k}, b_{k} \geq 0$ and $d_{k}>0, k=1,2,3, \ldots$, be constants. If

$$
\begin{aligned}
u(t) \leq & h(t)+\left(\int_{s_{m}}^{t}\left(\xi-s_{m}\right)^{\alpha-1} p(\xi) u(\xi) d \xi\right) \varphi(t) \\
& +\left(c_{\bar{m}} u\left(t_{\bar{m}}^{-}\right)+d_{\bar{m}} \int_{t_{\bar{m}}}^{t}\left(\xi-t_{\bar{m}}\right)^{\alpha-1} u(\xi) d \xi+b_{\bar{m}}\right) \psi(t), \quad t \in \mathbb{R}_{+},
\end{aligned}
$$

where $s_{m}$ and $t_{\bar{m}}$ are defined by (2.1), then we have

$$
\begin{aligned}
u(t) \leq & h(t)+e^{\int_{s_{m}}^{t} p(\xi)\left(\xi-s_{m}\right)^{\alpha-1} d \xi}\left\{\sum_{0<k \leq m}\left(\prod_{k<j \leq m} c_{j} G_{j}\right) H_{k}\left(b_{k}+c_{k} h\left(t_{k}^{-}\right)+d_{k} K_{k}\right)\right. \\
& \left.+\sum_{0<k \leq m}\left(\prod_{k<j \leq m} c_{j} G_{j}\right) c_{k} H_{k} \int_{s_{k-1}}^{t_{k}} p(\eta) h(\eta)\left(\eta-s_{k-1}\right)^{\alpha-1} e^{\int_{\eta}^{t_{k}} p(\xi)\left(\xi-s_{k-1}\right)^{\alpha-1} d \xi} d \eta\right\} \\
& +\int_{s_{m}}^{t} p(\eta) h(\eta)\left(\eta-s_{m}\right)^{\alpha-1} e^{\int_{\eta}^{t} p(\xi)\left(\xi-s_{m}\right)^{\alpha-1} d \xi} d \eta, \\
& t \in\left(s_{k}, t_{k+1}\right], k=0,1,2, \ldots,
\end{aligned}
$$


and

$$
\begin{aligned}
u(t) \leq & h(t)+e^{d_{\bar{m}} \frac{\left(t-t_{\overline{\bar{m}}}\right)^{\alpha}}{\alpha}}\left\{\sum_{0<k \leq \bar{m}}\left(\prod_{k<j \leq \bar{m}} c_{j} P_{j}\right)\left(b_{k}+c_{k} h\left(t_{k}^{-}\right)+d_{k} K_{k}\right)\right. \\
& \left.+\sum_{0<k \leq \bar{m}}\left(\prod_{k<j \leq \bar{m}} c_{j} P_{j}\right) c_{k} \int_{s_{k-1}}^{t_{k}} p(\eta) h(\eta)\left(\eta-s_{k-1}\right)^{\alpha-1} e^{\int_{\eta}^{t_{k}} p(\xi)\left(\xi-s_{k-1}\right)^{\alpha-1} d \xi} d \eta\right\}, \\
& t \in\left(t_{k}, s_{k}\right], k=1,2,3, \ldots,
\end{aligned}
$$

where the constants $K_{k}, k=1,2,3, \ldots$, are defined by $K_{k}=\int_{t_{k}}^{s_{k}}\left(\xi-t_{k}\right)^{\alpha-1} h(\xi) d \xi$.

Proof For $t \in \mathbb{R}_{+}$, setting

$$
\begin{aligned}
y(t)= & \left(\int_{s_{m}}^{t}\left(\xi-s_{m}\right)^{\alpha-1} p(\xi) u(\xi) d \xi\right) \varphi(t) \\
& +\left(c_{\bar{m}} u\left(t_{\bar{m}}^{-}\right)+d_{\bar{m}} \int_{t_{\bar{m}}}^{t}\left(\xi-t_{\bar{m}}\right)^{\alpha-1} u(\xi) d \xi+b_{\bar{m}}\right) \psi(t),
\end{aligned}
$$

we have

$$
{ }_{s_{m}} D^{\alpha} y(t)=p(t) u(t), \quad y\left(s_{0}\right)=0
$$

for $t \in\left(s_{k}, t_{k+1}\right], k=0,1,2, \ldots$, and

$$
y(t)=c_{\bar{m}} u\left(t_{\bar{m}}^{-}\right)+d_{\bar{m}} \int_{t_{\bar{m}}}^{t}\left(\xi-t_{\bar{m}}\right)^{\alpha-1} u(\xi) d \xi+b_{\bar{m}}
$$

for $t \in\left(t_{k}, s_{k}\right], k=1,2,3, \ldots$ Since $u(t) \leq h(t)+y(t), t \in \mathbb{R}_{+}$, this reduces to

$$
s_{m} D^{\alpha} y(t) \leq p(t) y(t)+p(t) h(t), \quad y\left(s_{0}\right)=0,
$$

and

$$
\begin{aligned}
y(t) \leq & c_{\bar{m}} y\left(t_{\bar{m}}^{-}\right)+d_{\bar{m}} \int_{t_{\bar{m}}}^{t}\left(\xi-t_{\bar{m}}\right)^{\alpha-1} y(\xi) d \xi \\
& +\left(b_{\bar{m}}+c_{\bar{m}} h\left(t_{\bar{m}}^{-}\right)+d_{\bar{m}} K_{\bar{m}}\right) .
\end{aligned}
$$

Now Theorem 2.1, together with the inequality $u(t) \leq h(t)+y(t)$, yields estimates (2.18) and (2.19), completing the proof.

Next, we obtain the following corollary by putting constant values $h(t) \equiv B>0$ and $p(t) \equiv M>0$.

Corollary 2.2 Let constants $c_{k}, b_{k} \geq 0$ and $d_{k}>0, k=1,2,3, \ldots$ If

$$
\begin{aligned}
u(t) \leq & B+\left(M \int_{s_{m}}^{t}\left(\xi-s_{m}\right)^{\alpha-1} u(\xi) d \xi\right) \varphi(t) \\
& +\left(c_{\bar{m}} u\left(t_{\bar{m}}^{-}\right)+d_{\bar{m}} \int_{t_{\bar{m}}}^{t}\left(\xi-t_{\bar{m}}\right)^{\alpha-1} u(\xi) d \xi+b_{\bar{m}}\right) \psi(t), \quad t \in \mathbb{R}_{+},
\end{aligned}
$$


where $s_{m}$ and $t_{\bar{m}}$ are defined by (2.1), then we have

$$
\begin{aligned}
u(t) \leq & B e^{M \frac{\left(t-s_{m}\right)^{\alpha}}{\alpha}}+e^{M \frac{\left(t-s_{m}\right)^{\alpha}}{\alpha}}\left\{\sum_{0<k \leq m}\left(\prod_{k<j \leq m} c_{j} G_{j}^{*}\right) H_{k} Z_{k}\right. \\
& \left.+B \sum_{0<k \leq m}\left(\prod_{k<j \leq m} c_{j} G_{j}^{*}\right) c_{k} H_{k}\left(e^{M \frac{\left(t_{k}-s_{k-1}\right)^{\alpha}}{\alpha}}-1\right)\right\} \\
& t \in\left(s_{k}, t_{k+1}\right], k=0,1,2, \ldots,
\end{aligned}
$$

and

$$
\begin{aligned}
u(t) \leq & B+e^{d_{\bar{m}} \frac{\left(t-t_{\bar{m}}\right)^{\alpha}}{\alpha}}\left\{\sum_{0<k \leq \bar{m}}\left(\prod_{k<j \leq \bar{m}} c_{j} P_{j}^{*}\right) Z_{k}\right. \\
& \left.+B \sum_{0<k \leq \bar{m}}\left(\prod_{k<j \leq \bar{m}} c_{j} P_{j}^{*}\right) c_{k}\left(e^{M \frac{\left(t_{k}-s_{k-1}\right)^{\alpha}}{\alpha}}-1\right)\right\}, \\
& t \in\left(t_{k}, s_{k}\right], k=1,2,3, \ldots,
\end{aligned}
$$

where $G_{j}^{*}$ and $P_{j}^{*}$ are defined as in Corollary 2.1, and $Z_{k}=b_{k}+B c_{k}+B d_{k}\left(s_{k}-t_{k}\right)^{\alpha} / \alpha$.

\section{Applications}

In this section, we establish two applications of noninstantaneous impulsive differential and integral inequalities. Let $J=[0, T]$ with $t_{n+1}=T$ and $\bar{J}=[0, \bar{T}]$ with $s_{n+1}=\bar{T}$ for some $n \geq 1$. The first purpose is accomplished by considering two problems that have the end points at $t_{n+1}$ and $s_{n+1}$, respectively. Now, we consider

$$
\left\{\begin{array}{l}
{ }_{s_{k}} D^{\alpha} u(t)-M u(t)+a(t) \leq 0, \quad t \in\left(s_{k}, t_{k+1}\right], k=0,1,2, \ldots, n, \\
u(t) \leq c_{k} u\left(t_{k}^{-}\right)+d_{k} \int_{t_{k}}^{t}\left(\xi-t_{k}\right)^{\alpha-1} u(\xi) d \xi, \quad t \in\left(t_{k}, s_{k}\right], k=1,2,3, \ldots, n \\
u(0)=u(T)+\lambda
\end{array}\right.
$$

and

$$
\left\{\begin{array}{l}
s_{k} D^{\alpha} v(t)-M v(t)+a(t) \leq 0, \quad t \in\left(s_{k}, t_{k+1}\right], k=0,1,2, \ldots, n, \\
v(t) \leq c_{k} v\left(t_{k}^{-}\right)+d_{k} \int_{t_{k}}^{t}\left(\xi-t_{k}\right)^{\alpha-1} v(\xi) d \xi, \quad t \in\left(t_{k}, s_{k}\right], k=1,2,3, \ldots, n+1 \\
v(0)=v(\bar{T})+\lambda
\end{array}\right.
$$

where $M>0, a(t) \in C\left[\mathbb{R}_{+}, \mathbb{R}_{+}\right], c_{k} \geq 0$, and $d_{k}>0$. Let us state the following conditions:

$\left(\mathrm{H}_{1}\right) e^{M \frac{\left(T-s_{n}\right)^{\alpha}}{\alpha}} \prod_{k=1}^{n} c_{k} G^{*}<1$,

$\left(\mathrm{H}_{2}\right) \quad \lambda \leq e^{M \frac{\left(T-s_{n}\right)^{\alpha}}{\alpha}} \int_{s_{n}}^{T} a(\eta)\left(\eta-s_{n}\right)^{\alpha-1} e^{-M \frac{\left(\eta-s_{n}\right)^{\alpha}}{\alpha}} d \eta$,

$\left(\mathrm{H}_{3}\right) \prod_{k=1}^{n+1} c_{k} G_{k}^{*}<1$,

$\left(\mathrm{H}_{4}\right) \quad \lambda \leq e^{d_{n+1} \frac{\left(\bar{T}-t_{n+1}\right)^{\alpha}}{\alpha}} \sum_{k=1}^{n+1}\left(\prod_{k<j \leq n+1} c_{j} P_{j}^{*}\right) c_{k} D_{k}$, where $D_{k}$ is defined by

$$
D_{k}=e^{M \frac{\left(t_{k}-s_{k-1}\right)^{\alpha}}{\alpha}} \int_{s_{k-1}}^{t_{k}} a(\eta)\left(\eta-s_{k-1}\right)^{\alpha-1} e^{-M \frac{\left(\eta-s_{k-1}\right)^{\alpha}}{\alpha}} d \eta, \quad k=1,2, \ldots, n+1
$$


Corollary 3.1 Let $u$ and $v$ be unknown functions satisfying (3.1) and (3.2), respectively. If $\left(\mathrm{H}_{1}\right)-\left(\mathrm{H}_{2}\right)$ hold, then $u(t) \leq 0$ for $t \in J$. If $\left(\mathrm{H}_{3}\right)-\left(\mathrm{H}_{4}\right)$ hold, then $v(t) \leq 0$ for $t \in \bar{J}$.

Proof Applying Theorem 2.1 to the first two inequalities in problem (3.1), we have

$$
\begin{aligned}
u(t) \leq & u(0) e^{M \frac{\left(t-s_{m}\right)^{\alpha}}{\alpha}} \prod_{0<k \leq m} c_{k} G_{k}^{*}-e^{M \frac{\left(t-s_{m}\right)^{\alpha}}{\alpha}} \sum_{0<k \leq m}\left(\prod_{k<j \leq m} c_{j} G_{j}^{*}\right) c_{k} H_{k} D_{k} \\
& -e^{M \frac{\left(t-s_{m}\right)^{\alpha}}{\alpha}} \int_{s_{m}}^{t} a(\eta)\left(\eta-s_{m}\right)^{\alpha-1} e^{-M \frac{\left(\eta-s_{m}\right)^{\alpha}}{\alpha}} d \eta, \\
& t \in\left(s_{k}, t_{k+1}\right], k=0,1,2, \ldots, n .
\end{aligned}
$$

Since $a(t) \geq 0$ for all $t \in \mathbb{R}_{+}$and all constants are positive, it is sufficient to show that $u(0) \leq 0$. At the end point $t=T$, we obtain

$$
\begin{aligned}
u(T) \leq & u(0) e^{M \frac{\left(T-s_{n}\right)^{\alpha}}{\alpha}} \prod_{k=1}^{n} c_{k} G_{k}^{*}-e^{M \frac{\left(T-s_{n}\right)^{\alpha}}{\alpha}} \sum_{k=1}^{n}\left(\prod_{k<j \leq n} c_{j} G_{j}^{*}\right) c_{k} H_{k} D_{k} \\
& -e^{M \frac{\left(T-s_{n}\right)^{\alpha}}{\alpha}} \int_{s_{n}}^{T} a(\eta)\left(\eta-s_{n}\right)^{\alpha-1} e^{-M \frac{\left(\eta-s_{n}\right)^{\alpha}}{\alpha}} d \eta .
\end{aligned}
$$

By conditions $\left(\mathrm{H}_{1}\right)-\left(\mathrm{H}_{2}\right)$ we have

$$
\begin{aligned}
u(0)\left(1-e^{M \frac{\left(T-s_{n}\right)^{\alpha}}{\alpha}} \prod_{k=1}^{n} c_{k} G_{k}^{*}\right) \leq & \lambda-e^{M \frac{\left(T-s_{n}\right)^{\alpha}}{\alpha}} \sum_{k=1}^{n}\left(\prod_{k<j \leq n} c_{j} G_{j}^{*}\right) c_{k} H_{k} D_{k} \\
& -e^{M \frac{\left(T-s_{n}\right)^{\alpha}}{\alpha}} \int_{s_{n}}^{T} a(\eta)\left(\eta-s_{n}\right)^{\alpha-1} e^{-M \frac{\left(\eta-s_{n}\right)^{\alpha}}{\alpha}} d \eta \\
\leq & 0,
\end{aligned}
$$

which yields $u(0) \leq 0$. Therefore $u(t) \leq 0$ for $t \in[0, T]$.

Next, we will show that $v(t) \leq 0$ for $t \in \bar{J}$. The application of Theorem 2.1 for the first two inequalities in problem (3.2) leads to

$$
\begin{aligned}
& v(t) \leq e^{d_{\bar{m}} \frac{\left(t-t_{\bar{m}}\right)^{\alpha}}{\alpha}}\left\{v(0) c_{\bar{m}} Q_{\bar{m}}^{*} \prod_{0<k<\bar{m}} c_{k} G_{k}^{*}-\sum_{0<k \leq \bar{m}}\left(\prod_{k<j \leq \bar{m}} c_{j} P_{j}^{*}\right) c_{k} D_{k}\right\}, \\
& t \in\left(t_{k}, s_{k}\right], k=1,2,3, \ldots, n+1 .
\end{aligned}
$$

Substituting the end point at $t=\bar{T}$, we have

$$
v(\bar{T}) \leq v(0) \prod_{k=1}^{n+1} c_{k} G_{k}^{*}-e^{d_{n+1} \frac{\left(\bar{T}-t_{n+1}\right)^{\alpha}}{\alpha}} \sum_{k=1}^{n+1}\left(\prod_{k<j \leq n+1} c_{j} P_{j}^{*}\right) c_{k} D_{k},
$$

which implies

$$
v(0)\left(1-\prod_{k=1}^{n+1} c_{k} G_{k}^{*}\right) \leq \lambda-e^{d_{n+1} \frac{\left(\bar{T}-t_{n+1}\right)^{\alpha}}{\alpha}} \sum_{k=1}^{n+1}\left(\prod_{k<j \leq n+1} c_{j} P_{j}^{*}\right) c_{k} D_{k}
$$$$
\leq 0,
$$ 
by conditions $\left(\mathrm{H}_{3}\right)-\left(\mathrm{H}_{4}\right)$. This means that $v(0) \leq 0$. In the same way, we can conclude that $v(t) \leq 0$ for $t \in \bar{J}$. The proof is completed.

Finally, we apply the noninstantaneous impulsive inequality to the initial value problem of the form

$$
\left\{\begin{array}{l}
s_{k} D^{\alpha} u(t)=f(t, u(t)), \quad t \in\left(s_{k}, t_{k+1}\right], k=0,1,2, \ldots \\
u(t)=c_{k} u\left(t_{k}^{-}\right)+d_{k} \int_{t_{k}}^{t}\left(\xi-t_{k}\right)^{\alpha-1} u(\xi) d \xi, \quad t \in\left(t_{k}, s_{k}\right], k=1,2, \ldots \\
u(0)=u_{0}
\end{array}\right.
$$

where $0<\alpha \leq 1, c_{k} \geq 0, d_{k}>0, u_{0} \in \mathbb{R}$, and the given function $f \in P C\left(U_{s_{k}} \times \mathbb{R}, \mathbb{R}\right)$ satisfies $\left(\mathrm{H}_{5}\right)|f(t, u)| \leq M|u|, M>0$, for all $t \in U_{s_{k}}$.

Corollary 3.2 If $\left(\mathrm{H}_{5}\right)$ holds, then the solution $u(t)$ of problem (3.3) is estimated as

$$
|u(t)| \leq e^{M \frac{\left(t-s_{m}\right)^{\alpha}}{\alpha}}\left|u_{0}\right| \prod_{0<k \leq m} c_{k} G_{k}^{*}, \quad t \in\left(s_{k}, t_{k+1}\right], k=0,1,2, \ldots
$$

and

$$
|u(t)| \leq e^{d_{\bar{m}} \frac{\left(t-t_{\bar{m}}\right)^{\alpha}}{\alpha}}\left|u_{0}\right| c_{\bar{m}} Q_{\bar{m}}^{*} \prod_{0<k<\bar{m}} c_{k} G_{k}^{*}, \quad t \in\left(t_{k}, s_{k}\right], k=1,2,3, \ldots
$$

Proof Taking the conformable fractional integral of order $\alpha$ to the first equation of problem (3.3), we obtain

$$
u(t)=u\left(s_{k}\right)+\int_{s_{k}}^{t}\left(\xi-s_{k}\right)^{\alpha-1} f(\xi, u(\xi)) d \xi, \quad t \in\left(s_{k}, t_{k+1}\right], k=0,1,2, \ldots
$$

From condition $\left(\mathrm{H}_{5}\right)$ it follows that

$$
\begin{aligned}
|u(t)| & \leq\left|u\left(s_{k}\right)\right|+\int_{s_{k}}^{t}\left(\xi-s_{k}\right)^{\alpha-1}|f(\xi, u(\xi))| d \xi \\
& \leq\left|u\left(s_{k}\right)\right|+M \int_{s_{k}}^{t}\left(\xi-s_{k}\right)^{\alpha-1}|u(\xi)| d \xi
\end{aligned}
$$

Since $u\left(s_{0}\right)=u_{0}$, by Theorem 2.2 inequalities (3.4)-(3.5) hold, and the proof is completed.

\section{Funding}

This research was funded by College of Industrial Technology, King Mongkut's University of Technology North Bangkok, Thailand.

\section{Availability of data and materials}

Data sharing not applicable to this article as no data sets were generated or analyzed during the current study.

\section{Competing interests}

The authors declare that they have no competing interests. 


\section{Author details}

'Department of Social and Applied Science, College of Industrial Technology, King Mongkut's University of Technology North Bangkok, Bangkok, Thailand. ${ }^{2}$ Department of Mathematics, University of loannina, loannina, Greece. ${ }^{3}$ Nonlinear Analysis and Applied Mathematics (NAAM) — Research Group, Department of Mathematics, Faculty of Science, King Abdulaziz University, Jeddah, Saudi Arabia. ${ }^{4}$ Department of Mathematics, Anand International College of Engineering, Jaipur, India. ${ }^{5}$ Intelligent and Nonlinear Dynamic Innovations Research Center, Department of Mathematics, Faculty of Applied Science, King Mongkut's University of Technology North Bangkok, Bangkok, Thailand.

\section{Publisher's Note}

Springer Nature remains neutral with regard to jurisdictional claims in published maps and institutional affiliations.

\section{Received: 7 April 2018 Accepted: 17 September 2018 Published online: 25 September 2018}

\section{References}

1. Podlubny, I.: Fractional Differential Equations. Academic Press, San Diego (1999)

2. Kilbas, A.A., Srivastava, H.M., Trujillo, J.J.: Theory and Applications of Fractional Differential Equations. North-Holland Mathematics Studies, vol. 204. Elsevier, Amsterdam (2006)

3. Diethelm, K.: The Analysis of Fractional Differential Equations. Lecture Notes in Mathematics. Springer, Berlin (2010)

4. Agarwal, R.P., Zhou, Y., He, Y.: Existence of fractional neutral functional differential equations. Comput. Math. Appl. 59, 1095-1100 (2010)

5. Ahmad, B., Ntouyas, S.K., Tariboon, J.: Existence results for mixed Hadamard and Riemann-Liouville fractional integro-differential equations. Adv. Differ. Equ. 2015, 293 (2015)

6. Ahmad, B., Nieto, J.J.: Riemann-Liouville fractional integro-differential equations with fractional nonlocal integral boundary conditions. Bound. Value Probl. 2011, 36 (2011)

7. Ahmad, B., Ntouyas, S.K., Alsaedi, A.: New existence results for nonlinear fractional differential equations with three-point integral boundary conditions. Adv. Differ. Equ. 2011, Article ID 107384 (2011)

8. Tariboon, J., Ntouyas, S.K., Thiramanus, P.: Riemann-Liouville fractional differential equations with Hadamard fractional integral conditions. Int. J. Appl. Math. Stat. 54, 119-134 (2016)

9. Ahmad, B., Ntouyas, S.K., Alsaedi, A.: A study of nonlinear fractional differential equations of arbitrary order with Riemann-Liouville type multistrip boundary conditions. Math. Probl. Eng. 2013, Article ID 320415 (2013)

10. Alghamdi, N., Ahmad, B., Ntouyas, S.K., Alsaedi, A.: Sequential fractional differential equations with nonlocal boundary conditions on an arbitrary interval. Adv. Differ. Equ. 2017, 246 (2017)

11. Ntouyas, S.K., Tariboon, J.: Fractional boundary value problems with multiple orders of fractional derivatives and integrals. Electron. J. Differ. Equ. 2017, 100 (2017)

12. Thaiprayoon, C., Ntouyas, S.K., Tariboon, J.: On the nonlocal Katugampola fractional integral conditions for fractional Langevin equation. Adv. Differ. Equ. 2015, 374 (2015)

13. Abdeljawad, T:: On conformable fractional calculus. J. Comput. Appl. Math. 279, 57-66 (2015)

14. Khalil, R., Al Horani, M., Yousef, A., Sababheh, M.: A new definition of fractional derivative. J. Comput. Appl. Math. 264, 65-70 (2015)

15. Usta, F., Sarikaya, M.: Some improvements of conformable fractional integral inequalities. Int. J. Anal. Appl. 14, 162-166 (2017)

16. Anderson, D.R., Ulness, D.J.: Results for conformable differential equations. Preprint

17. Atangana, A., Baleanu, D., Alsaedi, A.: New properties of conformable derivative. Open Math. 13, $889-898$ (2015)

18. Katugampola, U.: A new fractional derivative with classical properties. arXiv:1410.6535 [math.CA]

19. Usta, F.: Explicit bounds on certain integral inequalities via conformable fractional calculus. Cogent Math. 4, Article ID 1277505 (2017)

20. Lakshmikantham, V., Bainov, D.D., Simeonov, P.S.: Theory of Impulsive Differential Equations. World Scientific, Singapore (1989)

21. Benchohra, M., Henderson, J., Ntouyas, S.K.: Impulsive Differential Equations and Inclusions, vol. 2. Hindawi Publishing Corporation, New York (2006)

22. Samoilenko, A.M., Perestyuk, N.A.: Impulsive Differential Equations. World Scientific, Singapore (1995)

23. Hernández, E., O'Regan, D.: On a new class of abstract impulsive differential equations. Proc. Am. Math. Soc. 141, $1641-1649$ (2013)

24. Pierri, M., O'Regan, D., Rolnik, V.: Existence of solutions for semi-linear abstract differential equations with not instantaneous impulses. Appl. Math. Comput. 219, 6743-6749 (2013)

25. Fečkan, M., Wang, J.R., Zhou, Y.: Periodic solutions for nonlinear evolution equations with non-instantaneous impulses. Nonauton. Dyn. Syst. 1, 93-101 (2014)

26. Gautam, G.R., Dabas, J.: Mild solution for fractional functional integro-differential equation with not instantaneous impulse. Malaya J. Mat. 2(3), 428-437 (2014)

27. Pandey, D.N., Das, S., Sukavanam, N.: Existence of solution for a second-order neutral differential equation with state dependent delay and non-instantaneous impulses. Int. J. Nonlinear Sci. 18(2), 145-155 (2014)

28. Wang, J.R., Li, X.: Periodic BVP for integer/fractional order nonlinear differential equations with non-instantaneous impulses. J. Appl. Math. Comput. 46, 321-334 (2014)

29. Sood, A., Srivastava, S.K.: On stability of differential systems with noninstantaneous impulses. Math. Probl. Eng. 2015, Article ID 691687 (2015)

30. Li, P., Xu, C.: Boundary value problems of fractional order differential equation with integral boundary conditions and not instantaneous impulses. J. Funct. Spaces 2015, Article ID 954925 (2015)

31. Pierri, M., Henríquez, H.R., Prokopczyk, A.: Global solutions for abstract differential equations with non-instantaneous impulses. Mediterr. J. Math. 13, 1685-1708 (2016)

32. Agarwal, R.P., Hristova, S., O'Regan, D.: Noninstantaneous impulses in Caputo fractional differential equations and practical stability via Lyapunov functions. J. Franklin Inst. 354, 3097-3119 (2017) 
33. Agarwal, R.P., O'Regan, D., Hristova, S.: Monotone iterative technique for the initial value problem for differential equations with non-instantaneous impulses. Appl. Math. Comput. 298, 45-56 (2017)

34. Bai, L., Nieto, J.J., Wang, X.: Variational approach to non-instantaneous impulsive nonlinear differential equations. J. Nonlinear Sci. Appl. 10, 2440-2448 (2017)

35. Wang, J.R., Fečkan, M., Tian, Y.: Stability analysis for a general class of non-instantaneous impulsive differential equations. Mediterr. J. Math. 14, 46 (2017). https://doi.org/10.1007/s00009-017-0867-0

36. Yang, D., Wang, J.R.: Integral boundary value problems for nonlinear non-instantaneous impulsive differential equations. J. Appl. Math. Comput. 55, 59-78 (2017). https://doi.org/10.1007/s12190-016-1025-8

Submit your manuscript to a SpringerOpen ${ }^{\circ}$ journal and benefit from:

- Convenient online submission

$\checkmark$ Rigorous peer review

Open access: articles freely available online

- High visibility within the field

- Retaining the copyright to your article

Submit your next manuscript at $\gg$ springeropen.com 